\title{
Aparición paradójica de tuberculomas encefálicos durante el tratamiento de tuberculosis en pacientes inmunocompetentes
}

\author{
Jairo Lizarazo \\ Hospital Universitario Erasmo Meoz, Cúcuta, y Universidad de Pamplona, Pamplona, \\ Norte de Santander, Colombia.
}

\begin{abstract}
Se presentan dos casos de aparición de tuberculomas encefálicos durante el tratamiento de tuberculosis en pacientes inmunocompetentes. El primero, un hombre adulto que presentó una lesión frontal derecha que requirió cirugía y el segundo, una niña con múltiples lesiones, localizadas principalmente en el tallo cerebral. Los dos pacientes evolucionaron satisfactoriamente con el tratamiento. Esta paradójica presentación de la tuberculosis se atribuye a una rara interacción inmunológica entre el huésped y el bacilo tuberculoso. Los pacientes con esta patología no requieren cambio en el tratamiento antituberculoso y los esteroides son de utilidad en el alivio sintomático. La cirugía se reserva para aquellos casos de hipertensión endocraneana incontrolable o cuando hay incertidumbre diagnóstica. El pronóstico de esta patología es generalmente bueno.
\end{abstract}

Palabras clave: tuberculoma, sistema nervioso central, tuberculosis, inmunidad.

Paradoxical appearance of encephalic tuberculomas during treatment for tuberculosis in immunocompetent patients

\begin{abstract}
Two cases of appearance of encephalic tuberculomas during anti-tuberculous treatment in immunocompetent patients are presented. The first an adult man presenting a right frontal lesion which required surgical treatment and the second a girl with multiple lesions located mainly in the brainstem. This paradoxical presentation is attributed to a bizarre immunological reaction between the host and the tuberculosis bacillus. Patients with this condition do not require changes in anti-tuberculous treatment, and steroids are helpful in alleviating the symptoms. Surgery is indicated only for cases of uncontrollable intracranial hypertension or when there is diagnostic uncertainty. The prognosis of this entity is usually good.
\end{abstract}

Key words: tuberculoma, central nervous system, tuberculosis, immunity.

La meningitis tuberculosa es la forma de presentación más frecuente de la tuberculosis del sistema nervioso central (SNC) (1). Las otras formas de presentación son los tuberculomas, los abscesos tuberculosos y las lesiones espinales (1). Los tuberculomas encefálicos producen hipertensión endocraneana y signos focales neurológicos que ponen en peligro la vida del paciente (2). La aparición paradójica de tuberculomas encefálicos durante el curso del tratamiento

Correspondencia:

Jairo Lizarazo, Calle 13 Nº 1E-44, consultorio 404B, Cúcuta, Colombia.

Teléfono: 5722635 y 572 2652; fax: 5835161

jflizar@ telecom.com.co

Recibido: 11/08/03; aceptado: 22/02/04 de la tuberculosis, usualmente pulmonar o meníngea, es un reto diagnóstico y terapéutico (3). Se presentan dos casos con esta rara patología y se hace una revisión del tema.

\section{Caso 1}

Se trata de un paciente de 53 años de edad, procedente de Cúcuta, de ocupación conductor de vehículos que consultó el 01/08/91 a la Clínica del Instituto del Seguro Social por cuadro clínico de 15 días de evolución consistente en adinamia, decaimiento general y confusión mental.

Tenía el antecedente de tuberculosis pulmonar diagnosticada el 05/02/91 mediante baciloscopia positiva de esputo (++) y por la presencia de lesiones retículo-nodulares confluentes de 
predominio apical derecho en la radiografía de tórax. Le iniciaron tratamiento con cuatro drogas (isoniacida, rifampicina, estreptomicina y pirazinamida) con buena respuesta clínica. El 22/ 05/91 fue revisado en consulta externa en la cual se le encontró asintomático, con una baciloscopia en esputo negativa y refirió que estaba recibiendo el tratamiento antituberculoso. Cuando ingresó a la clínica estaba siendo tratado con isoniacida y rifampicina (segunda fase del tratamiento acortado supervisado de 6 meses).

En el examen físico se encontró un paciente en regulares condiciones generales con tensión arterial de $120 / 80 \mathrm{~mm} \mathrm{Hg}$, frecuencia cardiaca de 84 pulsaciones por minuto, frecuencia respiratoria de 20 por minuto y temperatura de $36,6^{\circ} \mathrm{C}$. La ausculatación cardiopulmonar fue normal y la palpación del abdomen fue negativa. El examen neurológico evidenció un paciente somnoliento, confuso, con lenguaje normal, leve hemiparesia izquierda, reflejos miotáticos aumentados en el lado izquierdo e incontinencia de esfínteres.

Los exámenes paraclínicos se consignan en el cuadro 1. La tomografía computarizada de cráneo mostró una lesión frontal derecha con extensión a través del cuerpo calloso con dos porciones redondeadas: una medial isodensa que captaba uniformemente el medio de contraste y otra, lateral, hipodensa con patrón de realce anular, importante edema vasógeno perilesional, efecto de masa y desviación de la línea media (figura 1).

El paciente se deterioró clínicamente; su nivel de conciencia se profundizó hasta el estupor; aparecieron reflejos patológicos de liberación frontal (prensión palmar bilateral, trompa y succión) y empeoró la hemiparesia izquierda. El enfermo se trató inicialmente con esteroides y se decidió llevar a cirugía para descomprimir el cerebro. El 12/08/91 se le practicó lobectomía del polo frontal derecho y resección total de la masa. Ésta, de $4 \times 3 \times 2 \mathrm{~cm}$, se encontraba encapsulada y se conformaba de dos partes redondeadas, una sólida y otra con una cavidad Ilena de material verdoso. Existía edema cerebral importante. La coloración de Zielh- Neelsen del material verdoso fue positiva para bacilos ácido alcohol resistentes (baar). El estudio histopatológico mostró un tejido cerebral destruido por formaciones granulo-

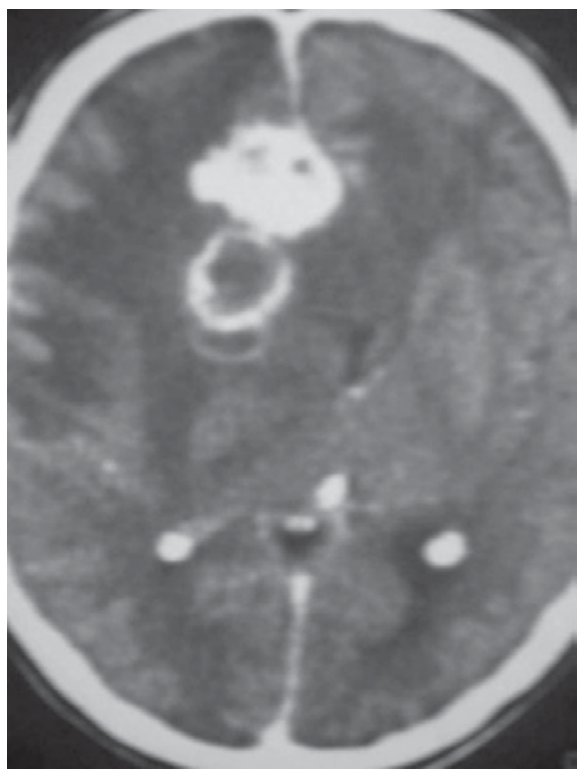

Figura 1. Tomografía de cráneo del caso 1 (02/08/91). Masa frontal derecha con extensión al cuerpo calloso con dos porciones redondeadas: una medial isodensa que capta uniformemente el medio de contraste y otra, lateral hipodensa, con patrón de captación anular. Se aprecia importante edema vasógeno perilesional, efecto de masa y desviación de la línea media.

Cuadro 1. Exámenes paraclínicos del caso 1.

\begin{tabular}{ll}
\hline Prueba & Resultado \\
\hline Cuadro hemático: & hemoglobina: $15,5 \mathrm{~g} \%$ \\
& leucocitos: $6.000 ;$ neutrófilos: $74 \%$; linfocitos: $21 \%$; eosinófilos: $4 \%$; monocitos, $1 \%$ \\
Química sanguínea: & glucemia: $87 \mathrm{mg} / \mathrm{dl}$; creatinina: $0,89 \mathrm{mg} / \mathrm{dl}$ \\
Parcial de orina: & normal \\
Radiografía de tórax: & lesión cicatricial pulmonar apical derecha \\
LCR: & incoloro, transparente; 2 linfocitos; proteínas: $36 \mathrm{mg} \% ;$ glucosa: $45 \mathrm{mg} \%$ \\
Tinciones: & Gram, negativo; ZN, negativo para baar \\
ELISA para VIH: & no reactivo \\
\hline
\end{tabular}


matosas, cuyos centros presentaban necrosis colicuativa, no caseosa y alrededor de este material existía proliferación de linfocitos, algunos histiocitos y fibroblastos. También, se observaron focos de polimorfonucleares. No se vieron células de Langhans. La coloración para baar fue positiva. No se hicieron cultivos. La evolución luego de la cirugía fue de una rápida mejoría sin déficit neurológico residual. El estudio del LCR descartó meningitis tuberculosa. Se le reinició tratamiento antituberculoso con cuatro drogas (isoniacida, rifampicina, estreptomicina y pirazinamida) y se continuaron los esteroides por 2 semanas. El 24/ 08/91 se le dio salida por mejoría. El 16/01/92 se controló por última vez en consulta externa; se encontraba asintomático con un examen físico y neurológico normal y estaba recibiendo isoniacida, rifampicina y etambutol; la baciloscopia en esputo era negativa. Se le indicó tratamiento antituberculoso por 1 año el cual concluyó sin novedades. Cuatro años después se le contactó por teléfono y se pudo establecer que seguía asintomático y vivía en Bogotá en donde trabajaba manejando un taxi.

\section{Caso 2}

Se trata de una paciente de sexo femenino de 10 años de edad, procedente del área rural de Cúcuta, estudiante de primaria que consultó el 28/07/02 al Hospital Erasmo Meoz por presentar cefalea crónica intermitente; en los últimos 6 días había tenido fiebre y vómito; desde el día anterior a su ingreso le notaron alteración del estado de conciencia, llanto inmotivado, irritabilidad y agitación psicomotora. Tenía antecedentes de aplicación de BCG en el periodo neonatal. No había historia de contacto tuberculoso. En 01/02 estuvo hospitalizada en la misma institución por un síndrome febril prolongado, cuya etiología no se pudo establecer. En los siguientes meses, la niña no había presentado síntomas.

En el examen físico de ingreso se encontró una niña en regulares condiciones generales; signos vitales: tensión arterial de 97/52 mm Hg, frecuencia cardíaca de 100 pulsaciones por minuto, frecuencia respiratoria de 28 porminuto $y$ temperatura axilar de $37,5^{\circ} \mathrm{C}$. Los ruidos cardiacos y respiratorios eran normales; el abdomen era blando y no se palparon masas ni megalias. El examen neurológico evidenció una niña alerta, confusa, que no obedecía órdenes y con un lenguaje escaso, pero bien articulado. Los pares craneanos fueron normales, inclusive el fondo de ojo. Tenía rigidez de nuca y signos meníngeos de Kernig y Brudzinski. No tenía déficit motor ni sensitivo en los miembros. Los reflejos miotáticos fueron simétricos 2/4 y controlaba los esfínteres. Por el síndrome meníngeo y la confusión mental, se le practicó una punción lumbar diagnóstica. En el cuadro 2 aparecen los resultados de los exámenes paraclínicos.

Inicialmente, se manejó con antibióticos (ceftriaxona y vancomicina) pensando en una meningitis piógena. En vista de su falta de mejoría se sospechó la etiología tuberculosa y el 12/08/ 02 se le inició tratamiento antituberculoso con cuatro medicamentos (isoniacida, rifampicina, estreptomicina y pirazinamida) y esteroides. La niña mejoró rápidamente y 10 días después se le dio salida. Durante su hospitalización, desarrolló una paresia bilateral del VI par con diplopía, único déficit anotado al egreso. Los resultados del LCR están consignados en el cuadro 3.

Cuadro 2. Resultados de los exámenes paraclínicos de la primera hospitalización del caso 1 (28/07/02-23/08/02).

\begin{tabular}{ll}
\hline Prueba & Resultado \\
\hline Cuadro hemático: & hemoglobina: $11,9 \mathrm{~g} \%$ \\
& leucocitos: $11.100 ;$ neutrófilos: $84 \%$; linfocitos $16 \%$; plaquetas: 302.000 \\
Velocidad de sedimentación globular: & $30 \mathrm{~mm}$ a la hora \\
Química sanguínea: & normal \\
Parcial de orina: & normal \\
TC de cráneo (29/07/02 y 13/08/02): & leve dilatación ventricular, aumento del realce meníngeo \\
Radiografía de tórax: & normal \\
Tuberculina (PPD): & 0 mm \\
ELISA para VIH: & no reactivo \\
\hline
\end{tabular}


Cuadro 3. Resultados del estudio del LCR del caso 2.

\begin{tabular}{lcccccc}
\hline Fecha & Color/aspecto & Células/ml & $\begin{array}{c}\text { Linfocitos } \\
\%\end{array}$ & $\begin{array}{c}\text { Glucosa } \\
\text { (mg/dl) }\end{array}$ & $\begin{array}{c}\text { Proteínas } \\
\text { (mg/d) }\end{array}$ & $\begin{array}{l}\text { Tinciones } \\
\text { (Gram, BK, } \\
\text { tinta china) }\end{array}$ \\
\hline $28 / 07 / 02$ & Incoloro/transparente & 60 & 92 & 8 & 225 & Negativas \\
$31 / 07 / 02$ & Incoloro/transparente & 120 & 90 & 7 & 203 & Negativas \\
$10 / 08 / 02$ & Amarillo/transparente & 48 & 90 & 11 & 270 & Negativas \\
$22 / 08 / 02$ & Incoloro/transparente & 90 & 95 & 31 & 52 & Negativas \\
\hline
\end{tabular}

Los cultivos para gérmenes comunes y BK fueron negativos.

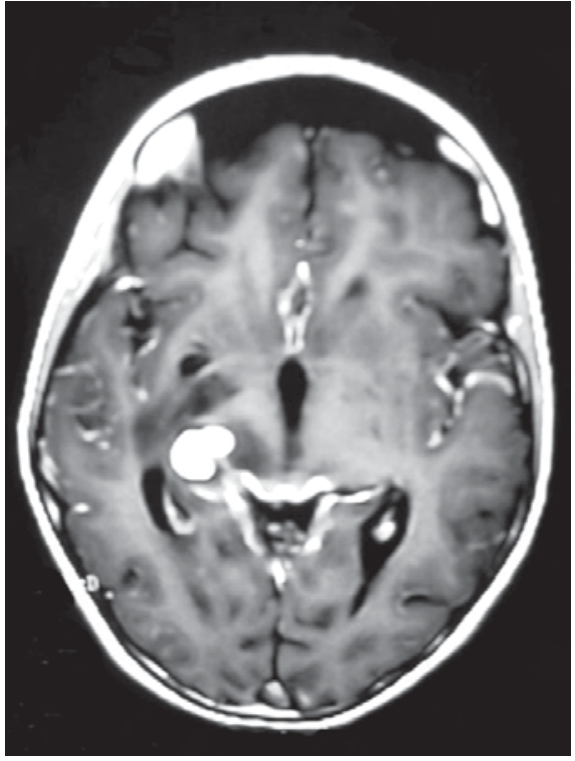

a

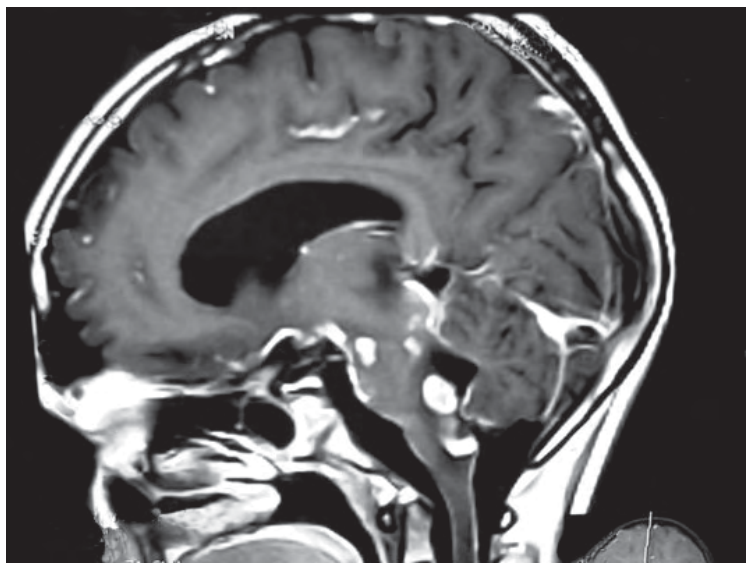

C

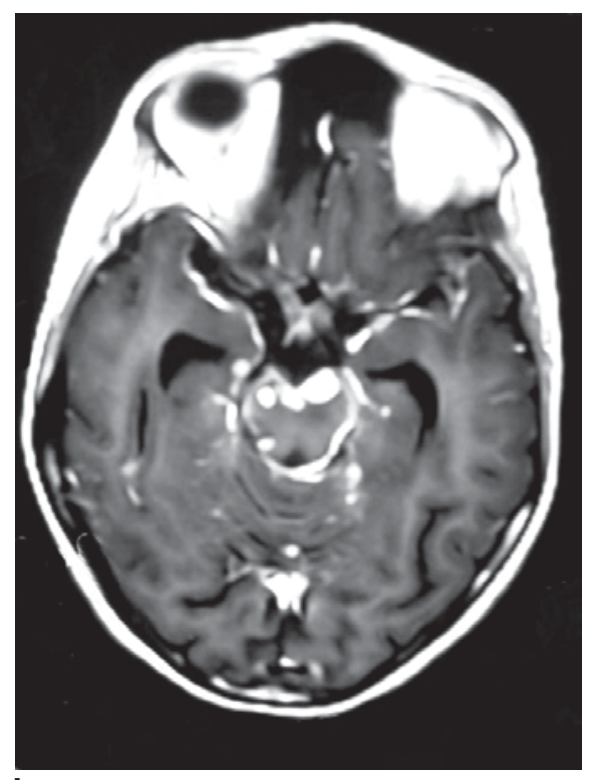

b

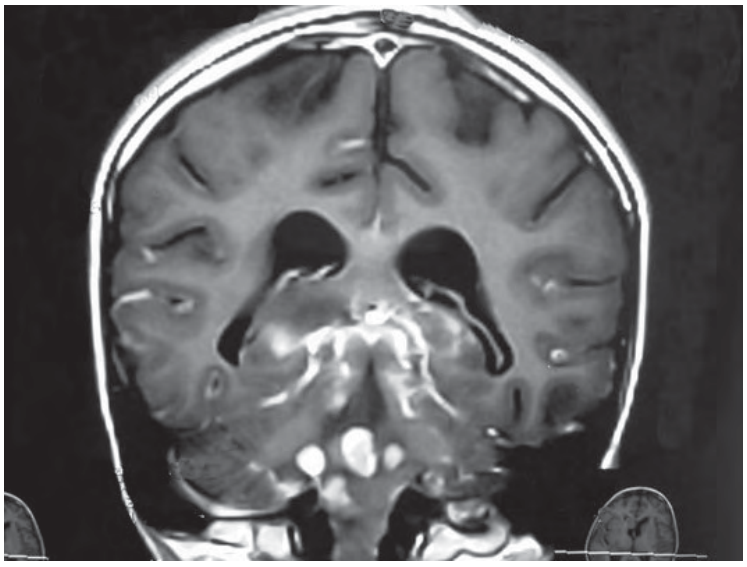

d

Figura 2. Resonancia magnética del caso 2 (20/11/02). a) Corte axial del T1 que muestra una lesión tálamo-capsular derecha que capta homogéneamente el gadolinio. b) Corte axial del T1 en el cual se ven múltiples lesiones mesencefálicas de predominio en los pedúnculos cerebrales con captación homogénea del medio de contraste. c) Corte sagital en el T1 que demuestra múltiples tuberculomas en el tallo cerebral. d) Corte coronal en el T1 que evidencia lesiones supra e infratentoriales. 
La niña siguió con tratamiento antituberculoso y se retiraron los esteroides en el mes siguiente; su cuadro clínico continuó sin cambios. En noviembre/02 nuevamente presentó fiebre y apareció una parálisis facial derecha y pérdida de la audición por el oído ipsilateral. El examen físico general era normal; el examen neurológico mostró una paciente alerta, con una esfera mental normal, lenguaje fluido, oftalmoplejía internuclear derecha, parálisis facial periférica derecha, sordera neurosensorial derecha y ataxia troncular.

Ante los nuevos hallazgos, la niña fue hospitalizada y se le practicó una resonancia magnética de cerebro que mostró múltiples tuberculomas en el tallo cerebral y la región tálamocapsular derecha (figura 2).

Se decidió dejar tratamiento antituberculoso con tres drogas (isoniacida, rifampicina y pirazinamida) y se reiniciaron esteroides (prednisona). En 01/ 03 , al suspender los esteroides, hubo empeoramiento del cuadro clínico, el cual consistió en disminución de la agudeza visual asociado con atrofia de los discos ópticos, cefalea y hemiparesia izquierda. Se reiniciaron los esteroides (prednisona, $1 \mathrm{mg} / \mathrm{kg}$ por día) y se le practicó una tomografía de cráneo que no mostró cambios de las lesiones encefálicas. La niña fue mejorando paulatinamente de la hipertensión endocraneana y de la hemiparesia izquierda y la tomografía del 24/04/03 mostró desaparición de los tuberculomas, atrofia cerebral y dilatación ventricular leves y una pequeña lesión hipodensa residual lentículo-capsular derecha (figura 3). La evaluación neurológica del 15/06/03 evidenció atrofia óptica bilateral con leve disminución de la agudeza visual, oftalmoplejía internuclear derecha en mejoría, paresia facial periférica derecha, hipoacusia neurosensorial derecha y ataxia de tronco moderada. Se le suspendieron los esteroides y se le dejó tratamiento antituberculoso el cual llevó a cabo por un año. En la última consulta del 25/09/03 se encontró el mismo déficit neurológico.

\section{Discusión}

Los tuberculomas son masas granulomatosas, esféricas, firmes y avasculares que miden alrededor de 2 a $8 \mathrm{~cm}$ de diámetro. Están bien

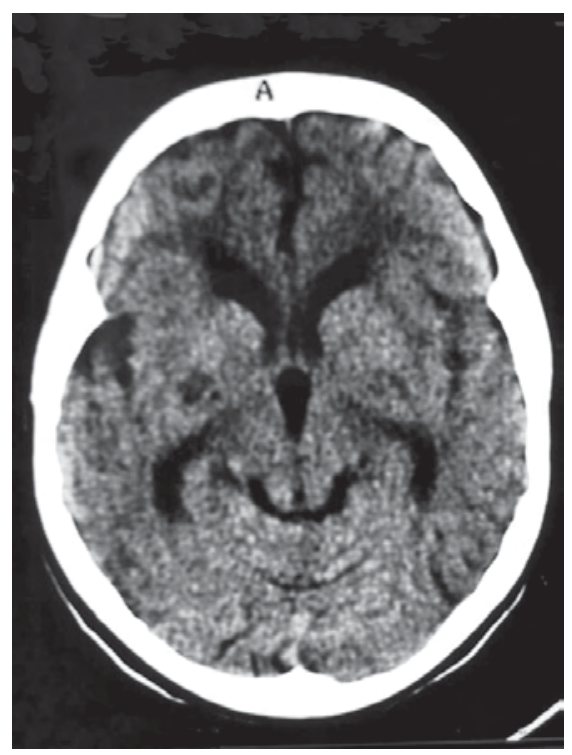

Figura 3. Tomografía de cráneo del caso 2 después del tratamiento (24/04/03). Corte a la altura de los núcleos basales: desaparición del tuberculoma; cambios de atrofia cerebral y dilatación ventricular leves; pequeña lesión residual lentículo-capsular derecha.

delimitados del tejido cerebral vecino el cual se encuentra comprimido alrededor de la lesión y revela edema y gliosis. En su interior, pueden contener áreas necróticas compuestas de material caseoso, ocasionalmente espeso y purulento, en el cual se pueden demostrar los bacilos tuberculosos (2), como se describió en el caso 1. Se deben diferenciar de los abscesos tuberculosos los cuales están formados por una colección encapsulada de pus que contiene bacilos tuberculosos viables (4-6). No obstante, algunos autores consideran a los tuberculomas y a los abscesos tuberculosos como variantes histológicas del mismo proceso patológico $(5,7)$.

Los tuberculomas intracraneanos son poco frecuentes en los países desarrollados, pero constituyen el $5 \%$ al $30 \%$ de todas las masas intracraneanas de los pacientes de algunos países en vías de desarrollo (8). En Colombia, Trujillo et al. informaron 13 casos en un solo centro hospitalario durante un período de 17 años (9). En una serie de 165 casos, Vergara et al. encontraron en $20 \%$ de los casos, tuberculomas del SNC mayores de $1 \mathrm{~cm}$ (10). También, se ha reportado un caso de absceso cerebral tuberculoso (11). 
Estas lesiones se presentan a cualquier edad, aunque son más frecuentes en niños y adultos jóvenes. En los primeros, tienden a localizarse en la fosa posterior, mientras que en los segundos su localización es preferentemente supratentorial (3).

Se cree que los tuberculomas intracraneanos son el resultado de la diseminación hematógena de la infección desde otra parte del cuerpo. El bacilo tuberculoso, una vez en el cerebro, lleva a la formación de tubérculos con caseificación central y células epitelioides y gigantes. Estos focos microscópicos son usualmente perivasculares y múltiples y gradualmente se alargan para formar numerosos tuberculomas macroscópicos pequeños que pueden fusionarse más tarde (12).

La aparición o el agrandamiento paradójico de los tuberculomas encefálicos durante el curso del tratamiento de la tuberculosis es un hecho poco frecuente. En una revisión de la literatura en inglés que abarcó 25 años sólo se encontraron 41 casos (24 de agrandamiento y 17 de aparición de tuberculomas) (3). En otra revisión de la literatura mundial, se reportaron 34 casos de aparición de tuberculomas durante el tratamiento antituberculoso (13). En los últimos años se ha descrito el mismo fenómeno tanto en pacientes inmunocompetentes (14-22) como en pacientes VIH positivos (23). En 1955, Choremis et al. informaron que a los niños a quienes se les iniciaba terapia antituberculosa ocasionalmente desarrollaban exacerbación de la fiebre y de los hallazgos patológicos radiológicos. Se han descrito las siguientes respuestas paradójicas durante el tratamiento de la tuberculosis tanto en pacientes negativos para VIH como en pacientes con sida: recrudescencia de la fiebre, agrandamiento de adenopatías, empeoramiento de los infiltrados pulmonares, derrame pleural, ascitis, nuevos o agrandamiento de tuberculomas y abscesos tuberculosos del SNC y síndrome de la vena cava superior $(24,25)$.

Se han postulado dos hipótesis sobre el desarrollo o el agrandamiento de los tuberculomas. La primera atribuye ese fenómeno a una disminución en la penetración de las drogas antituberculosas en el SNC. Esto es poco probable, si se tiene en cuenta que la isoniacida y la pirazinamida, fármacos usados en su tratamiento, atraviesan la barrera hematoencefálica en ausencia de inflamación. La teoría mencionada tampoco explicaría el porqué muchos de estos pacientes mejoran con sólo mantenerles la misma terapia antituberculosa que venían recibiendo. Otro argumento en su contra es el agrandamiento de los ganglios linfáticos en pacientes con linfadenitis tuberculosa que están recibiendo tratamiento etiológico y el desarrollo del síndrome de dificultad respiratoria del adulto en algunos pacientes que están siendo tratados para tuberculosis pulmonar. La segunda teoría, considera que se trata de una respuesta paradójica posiblemente asociada con una recuperación de la respuesta inmune al disminuir la carga del antígeno (26).

En los pacientes positivos para VIH, la mejoría del estado inmunológico después del inicio de la terapia antirretroviral de alta efectividad (HAART) o su intensificación con un inhibidor de la proteasa es el factor precipitante de lo que se ha denominado el síndrome de reconstitución inmunológica (27). Además de la aparición de tuberculomas o abscesos cerebrales tuberculosos, también se han descrito la aparición de retinitis por citomegalovirus, linfadenitis por el complejo Mycobacterium avium, desarrollo de brote por herpes zóster, rápido empeoramiento de las lesiones del sarcoma de Kaposi y la aparición de múltiples lesiones cerebrales compatibles con leucoencefalopatía multifocal progresiva (28).

Narita et al. informaron la reacción paradójica a la iniciación del tratamiento antituberculoso en 12 $(36 \%)$ de 33 pacientes positivos para $\mathrm{VIH}$ que concomitantemente iniciaron HAART, en 2 (7\%) de 28 pacientes positivos para VIH tratados para tuberculosis antes del advenimiento de la HAART y en $1(2 \%)$ de 55 pacientes negativos para VIH que comenzaron quimioterapia tuberculostática (28).

La aparición o el agrandamiento de los tuberculomas encefálicos durante el tratamiento etiológico ocurre principalmente en personas jóvenes sin un claro predominio de género. Las lesiones aparecen entre 2 semanas y 27 meses después de iniciados los medicamentos antituberculosos $y$, 
usualmente, dichas lesiones se hicieron evidentes cuando la tuberculosis sistémica estaba siendo tratada con éxito, tal y como ocurrió en nuestros dos casos. Las manifestaciones clínicas corresponden a la localización de las lesiones y, frecuentemente, cursan con el síndrome de hipertensión endocraneana (3).

La tomografía de cráneo y la resonancia de cerebro son los estudios que más nos ayudan en el diagnóstico inicial de las masas intracraneanas. En la tomografía simple de cráneo, los tuberculomas cerebrales pueden ser isodensos o ligeramente hiperdensos; con la administración del medio de contraste, el patrón de realce de las lesiones puede ser homogéneo, en forma de anillo o una combinación de los anteriores. A veces, aparecen como lesiones con realce anular que tienen un área central hiperdensa o una calcificación (signo de la diana) (29). La tomografía de cráneo también ayuda a demostrar la meningitis basilar y la hidrocefalia; detecta el edema cerebral y predice el riesgo de herniación y sirve para supervisar la respuesta al tratamiento (3). En ocasiones, los hallazgos de la tomografía no son típicos y, como en el caso 1 , pueden simular un glioma del cuerpo calloso (30).

En la resonancia, los tuberculomas son típicamente isointensos con el cerebro en la imágenes ponderadas en el T1 y realzan notoriamente con la administración del gadolinio. En las imágenes ponderadas en el T2, los tuberculomas tienen una hiperdensidad central y un anillo hipointenso. Las imágenes hipointensas en el T2 están asociadas con aumento de la fibrosis, gliosis e infiltración de macrófagos (29). La resonancia es superior a la tomografía para demostrar la extensión de la lesión, especialmente en la fosa posterior y en el tallo cerebral bajo. En el $10 \%$ al $30 \%$ de los casos existen múltiples tuberculomas (3).

El diagnóstico diferencial de los tuberculomas se hace con otras enfermedades granulomatosas como las micosis y la sarcoidosis; con enfermedades parasitarias como la cisticercosis o la toxoplasmosis, y con neoplasias como los gliomas o las metástasis. En pacientes con sida puede ser necesaria una biopsia de las lesiones accesibles para excluir otras lesiones tratables o para evitar terapias empíricas innecesarias $(1,3)$.

El aislamiento de M. tuberculosis del LCR o de otro sitio ayuda en el diagnóstico, al igual que una prueba de tuberculina positiva o la historia de contacto con un paciente tuberculoso. En pacientes inmunocompetentes con meningitis tuberculosa, el BK es observado en el examen directo del LCR en el $5 \%$ al $85 \%$ de los adultos y en el 0 al $6 \%$ de los niños y se logra su cultivo en el $40 \%$ al $85 \%$ de los primeros y en el $35 \%$ al $85 \%$ de los últimos (1). En nuestro medio, la sensibilidad del examen directo y del cultivo del LCR en pacientes adultos inmunocompetentes con meningitis tuberculosa es del $9 \%$ y del $32 \%$, respectivamente (31).

La terapia óptima de los tuberculomas y de la meningitis tuberculosa es desconocida debido a la falta de estudios controlados (32). Las terapias usadas son extrapoladas de la tuberculosis pulmonar en donde sí existen estudios controlados. Los pacientes con tuberculomas deberían ser tratados empíricamente con, al menos, cuatro drogas (isoniacida, rifampicina, pirazinamida y estreptomicina), por lo menos, 2 meses. Luego, se completa el tratamiento con isoniacida y rifampicina por un mínimo de 12 meses $(3,32)$. En casos especiales, según la evolución clínica y de las imágenes diagnósticas, se hace necesario prolongar el tratamiento (13). Tampoco existen estudios controlados sobre la eficacia de los esteroides en esta patología, aunque parecen ser efectivos en el alivio de los síntomas de los pacientes con edema cerebral y déficit neurológico focal $(3,13)$. No obstante, los esteroides no previenen la aparición ni el agrandamiento de los tuberculomas. Se recomienda el uso de los esteroides, basados en los buenos resultados obtenidos en estudios no controlados de pacientes con meningitis tuberculosa, en los cuales se demostró disminución de la mortalidad y de la incidencia de secuelas neurológicas permanentes (7). Los esteroides se administran durante un período de 4 a 8 semanas (3).

La cirugía se reserva para cuando la terapia médica ha fallado en el alivio de la hipertensión 
endocraneana. La terapia antituberculosa siempre se debe dar a los pacientes operados debido al riesgo de diseminación de los bacilos a las meninges y la consiguiente meningitis tuberculosa. Los procedimientos quirúrgicos empleados en estos pacientes son la derivación del LCR debido a la hidrocefalia y la escisión, resección parcial o drenaje de los tuberculomas (3).

El pronóstico de estos pacientes es generalmente bueno cuando se detectan tempranamente y se tratan oportunamente. La mortalidad reportada es cercana al $10 \%$, una tercera parte queda con secuelas y un poco más de la mitad se recupera completamente $(3,13)$.

Es necesario enfatizar que casi todos los pacientes van a mejorar con el tratamiento. Es poco probable que la aparición o el agrandamiento de los tuberculomas sea debido a falla terapéutica. Por tanto, no hay indicación para cambiar de terapia. Los efectos antiinflamatorios de los esteroides disminuyen los síntomas neurológicos y pueden mejorar el resultado. La cirugía se debe reservar para aquellos casos de hipertensión endocraneana inmanejable médicamente o cuando el diagnóstico es incierto.

\section{Agradecimientos}

A Rafael Fandiño por su participación en el manejo neuroquirúrgico del caso 1; a Patricia Gutiérrez por su ayuda en el procesamieno de las imágenes diagnósticas y a Elizabeth Castañeda por sus valiosas sugerencias.

\section{Referencias}

1. García-Monco JC. Central nervous system tuberculosis. Neurol Clin 1999;17:737-59.

2. Garg RK. Tuberculosis of the central nervous system. Postgrad Med J 1999;75:133-40.

3. Afghani B, Lieberman JM. Paradoxical enlargement or development of intracranial tuberculomas during therapy: case report and review. Clin Infect Dis 1994;19:1092-9.

4. Whitener DR. Tuberculous brain abscess. Report of a case and review of the literature. Arch Neurol 1978;35:148-55.

5. Kumar R, Pandey CK, Bose N, Sahay S. Tuberculous brain abscess: clinical presentation, pathophysiology and treatment (in children). Child's Nerv Syst 2002;18:118-23.
6. Tyson G, Newman P, Strachan WE. Tuberculous brain abscess. Surg Neurol 1978;10:323-5.

7. Sheller JR, Des Prez RM. CNS tuberculosis. Neurol Clin 1986;4:143-58.

8. Gropper MR, Schulder M, Duran HL, Wolansky L. Cerebral tuberculosis with expansion into brainstem tuberculoma. J Neurosurg 1994;81:927-31.

9. Trujillo J, Toro G, Corso C. Tuberculomas cerebrales. Rev Fac Med UN Colombia 1971;37:412-6.

10. Vergara I, Saravia J, Toro G, Calderón A, Román G, Navarro de Román L. La infección tuberculosa del sistema nervioso central. Acta Med Colomb 1976;1:3352.

11. Palma RR, Lizarazo JF. Absceso cerebral tuberculoso. Acta Neurol Colomb 1987;3:16-9.

12. Lees AJ, MacLeod AF, Marshall J. Cerebral tuberculomas developing during treatment of tuberculous meningitis. Lancet 1980;1:1208-11.

13. Hejazi N, Hassler W. Multiple intracranial tuberculomas with atypical response to tuberculostatic chemotherapy: literature review and a case report. Infection $1997 ; 25: 233-9$

14. Chambers ST, Hendrickse WA, Record C, Rudge P, Smith H. Paradoxical expansion of intracranial tuberculomas during chemotherapy. Lancet 1984;2:181-4.

15. Teoh R, Humphries MJ, O'Mahony G. Symptomatic intracranial tuberculoma developing during treatment of tuberculosis: a report of 10 patients and review of literature. Q J Med 1987;63:449-60.

16. AbdulJabbar M. Paradoxical response to chemotherapy for intracranial tuberculoma: two case reports from Saudi Arabia. J Trop Med Hyg 1991;94:374-6.

17. Rao GP, Nadh BR, Hemaratnan A, Srinivas TV, Reddy PK. Paradoxal progression of tuberculous lesions during chemotherapy of central nervous system tuberculosis: report of 4 cases. J Neurosurg 1995;83:359-62.

18. Reiser M, Fatkenheuer G, DiehI V. Paradoxical expansion of intracranial tuberculomas during chemotherapy. J Infect 1997;35:88-90.

19. Andrade AS Filho, Gomes AG, Lemos ACM, Neves MC, Souza YMA, et al. Expansao paradoxal de lesoes de tuberculose cerebral durante 0 uso de tuberculostáticos. Arq Neuropsiquiatr 1999;57:471-5.

20. Pimentel MLV, Alves SMV, Novis SAP, Brandao RZ, Neto EB. Múltiplos tuberculomas intracerebrais na vigência de terapia específica para tuberculose pulmonar. Arq Neuropsiquiatr 2000;58:572-7.

21. Castro-Vilanova MD, Morín MM, Cantón R, Domingo J, Corral E, Rodríguez-García E, et al. Tuberculomas intracraneales en un paciente inmunocompentente. Rev Neurol 2000;31:96. 
22. Serrano M, Campistol J, Chávez B, Caritg J, Fortuny C, Costa JM. Tuberculomas intracraneales múltiples en la infancia. Rev Neurol 2001;33:44-6.

23. Ramdas K, Minamoto GY. Paradoxical presentation of intracranial tuberculomas after chemotherapy in a patient with AIDS. Clin Infect Dis 1994;19:793-4.

24. Eyer-Silva WA, Pinto JFC, Árabe J, Morais-de-Sá CA. Paradoxical reaction to the treatment of tuberculosis uncovering previously silent meningeal disease. Rev Soc Bras Med Trop 2002;35:59-61.

25. Vidal JE, Cimerman S, Shiavon Nogueira $\mathbf{R}$, Bonasser Filho F, Sztajnbok J, Marques da Silva PR, et al. Paradoxical reaction during treatment of tuberculosis brain abscess in a patient with AIDS. Rev Inst Med Trop S Paulo 2003;45:177-8.

26. Bekker LG, Maartens G, Steyn L, Kaplan G. Selective increase in plasma tumor necrosis factor-alpha and concomitant clinical deterioration after initiating therapy in patients with severe tuberculosis. J Infect Dis 1998;178:580-4.

27. Cheng VCC, Yuen K, Chan W, Wong SSY, Ma ESK, Chan RMT. Immunorestitucion disease involving the innate and adaptative response. Clin Infect Dis 2000;30:882-92.

28. Narita M, Ashkin D, Hollender E, Pitchenik AE. Paradoxical worsening of tuberculosis following antiretroviral therapy in patients with AIDS. Am J Respir Crit Care Med 1998;158:157-61.

29. Daniel RT, Henry PT, Rajshekhar V. Unusual MR presentation of cerebral parenchymal tuberculosis. Neurology India 2002;50:210-1.

30. Fath-Ordoubadi F, Lane RJM, Richards PG. Histological surprise: callosal tuberculoma presenting as malignant glioma. J Neurol Neurosurg Psychiatry 1997; 63:98-9.

31. Palma R, Lizarazo J, Vergara I, Toro G, Saravia J, Parra L, et al. La infección tuberculosa del sistema nervioso central en el Centro Hospitalario San Juan de Dios de Bogotá (1975-1986). Estudio de 86 casos. Acta Med Colomb 1988;13:106-24.

32. Thwaites G, Chau TTH, Mai NTH, Drobniewski F, McAdam K, Farrar J. Tuberculous meningitis. J Neurol Neurosurg Psychiatry 2000;68:289-99.

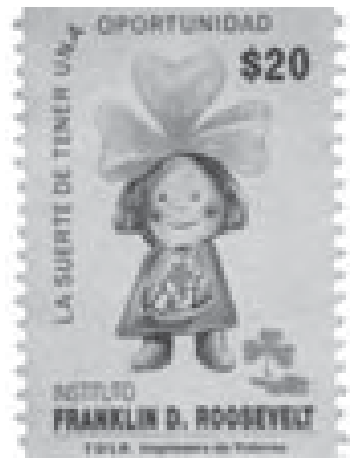

